\title{
Simultaneous discrimination: Parameters of reinforcement and ITI'
}

GERALD B. BIEDERMAR

Varying the duration of reinforcement and the duration of intertrial interval had no effect on the course of discrimination learning.

Simultaneous discrimination may be effectively studied in Skinner box situations which use a trial by trial procedure. In simultaneous discrimination the positive stimulus (S+-the stimulus correlated with reinforcement) and the negative stimulus ( $\mathrm{S}-$-the stimulus correlated with non-reinforcement) are present on each trial. In the case of pigeons, pecking the key that displays S+ produces reinforcement; in the case of rats depressing the bar related to $\mathrm{S}_{+}$ results in reinforcement (Biederman, 1967a, b).

Classically, learning theorists have placed considerable emphasis on amount of reinforcement as a facilitator of learning. Skinner box experiments typically use a reinforcement duration of 2 to $3 \mathrm{sec}$. The rationale behind the selection of these values is not clear. One purpose of the present experiment was to provide parametric evidence that might suggest the optimal reinforcement duration for discrimination training in a Skinner box.

Recent evidence has indicated that as learning progresses in discriminative training, S's responding is controlled by S+ (Terrace, 1967). The consequence of a response to $\mathrm{S}-$ (an error) is an intertrial timeout interval. Increasing the intertrial interval (ITI) should have decreasing importance for performance as training proceeds. Thus, a second purpose of this experiment was to study the effect of parameters of ITI on discriminative performance.

\section{Method}

The Ss were 36 white Carneaux male pigeons, experimentally naive at the start of training, maintained at 80 percent of their ad lib weight. Training took place in commercial operant conditioning chambers, automatically controlled. Stimuli were provided by standard stimulus projectors which illuminated the response keys. Pigeons were shaped on Day 1 to peck a key illuminated with white light for food reward in a single-key chamber. On Day 2 they were transferred to two-key experimental chambers where they were trained to respond to the lighted key. After a reinforced response, the illumination shifted on a random basis to one of the two alternate keys. No reinforcement was given for a response to a darkened key; this procedure obviated subject side-preference. On Days 3-5, 150 discrimination trials were given each day. The pairs of possible discriminanda are shown in Fig. 1. For each $\mathrm{S}$ two pairs of stimuli were selected for training; one pair received 100 training trials each day while the remaining pair received 50 training trials. ${ }^{2}$ Stimuli were counterbalanced with respect to positivity and amount of training across Ss. Color stimuli were not presented with figure stimuli, nor were Discriminations 4 and 5 presented together. On each trial the problem and the key displaying St were predetermined by "plug board" units programmed via random number sequences. Ss were assigned to reinforcement duration and ITI groups at random. Four Ss were assigned to each group in a 3 by 3 design with repeated measurements. The reinforcement durations were 1.5, 3 , or $6 \mathrm{sec}$, which represents the length of time the food-hopper was

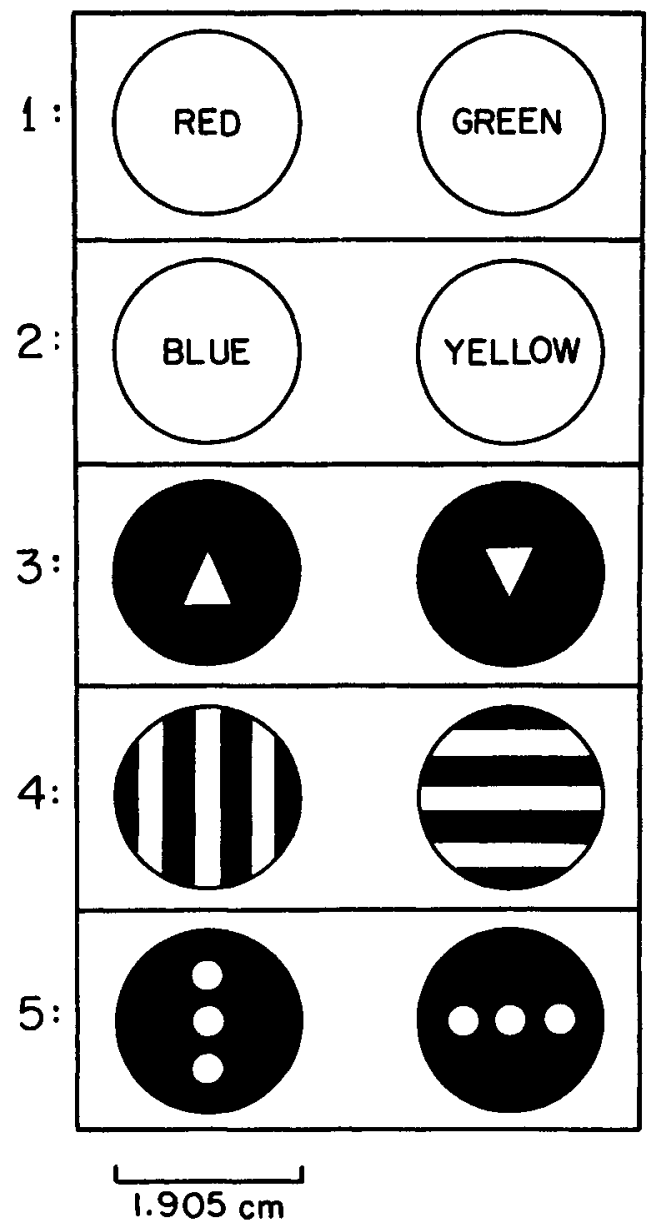

Fig. 1. The discriminative stimuli. 


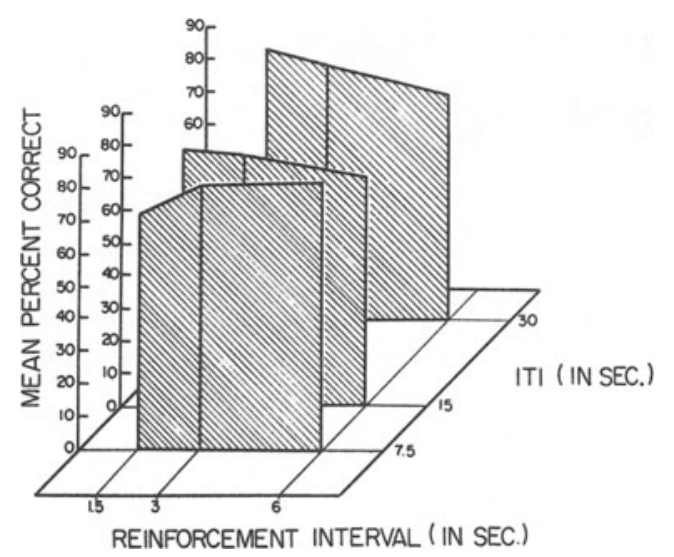

Fig. 2. Discrimination as a function of reinforcement duration and ITI.

available to $S$ following a correct response. The ITI durations were $7.5,15$, or 30 sec. During ITI the keys were darkened and deactivated. On a given trial the St and S- would appear. S's choice would either be followed by reinforcement or ITI of predetermined duration. The duration of ITI following a correct response was equal to the difference between that S's ITI and reinforcement durations. Thus for Ss with a 3-sec reinforcement duration and a 15-sec ITI, the ITI following a correct response was $12 \mathrm{sec}$. Resulis and Discussion

Inspection of Fig. 2 shows that there was no clear effect of either reinforcement duration or ITI when the data are plotted across blocks. Analysis of variance (with nine blocks of 50 trials) shows no significant main effect except for Blocks ( $F=35.34$, df $=$ $8 / 216, p<.005)$. For Reinforcement Durations and ITI main effects, $F<1$, df $=2 / 27$, respectively; Reinforcement Duration by ITI, $F=1.18$, df $=4 / 27, p>.25$. The interaction of Reinforcement Duration by Blocks, and ITI by Blocks were also insignificant, $F<1, \mathrm{df}=$ $16 / 26$, respectively, as was the triple interaction of Reinforcement Duration by ITI by Blocks ( $\mathrm{F}<1$, df = $32 / 216$ ).

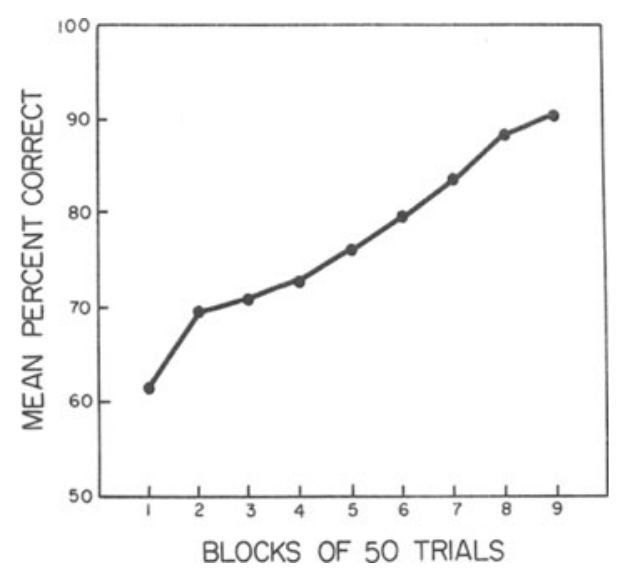

Fig. 3. Leaming as a function of blocks of 50 trials.

The results indicate that learning in the present procedure was independent of the amount of reward or the duration of ITI. The course of discrimination may be seen in Fig. 3, which illustrates a typical learning function.

This experiment points to the possibility that some standard experimental routines may be little more than superstitious behavior. In any event, the effective limits of ITI and reinforcement duration should be ascertained for the sake of both methodology and theory.

\section{References}

Biedeman, G. B. The overlearning reversal effeet: A function of the non-monotonicity of $\mathrm{S}-$ during discriminative training. Psychon. Sci., 1967a, 7, 385-386.

Biedeman, G. B. Stimulus function in simultaneous discrimination. Publication pending, 1967b.

Terrace, H. S. Discrimination learning and inhibition. Science, $1967,154,1677-1680$.

\section{Notes}

1. This research was partly supported by NRC grant APA 185 . I thank Miss M. Archer and Mr. G. Heighington for their assistance.

2. The purpose of using two discriminations of unequal training is irrelevant for the present study. All data presented here are in terms of combined performance on the two discriminations. 